\title{
Construction and analysis of Sudoku square designs with rectangles
}

\begin{abstract}
The present paper deals with the introduction of new experimental designs namely "Sudoku Square Designs with Rectangles". The construction and analysis of the Sudoku square designs with rectangles are explained in detail. The steps for the construction of proposed designs are illustrated with the help of a numerical example. Some applications of Sudoku designs with rectangles in real life situations are also discussed.
\end{abstract}

Keywords: latin squares, sequential method, rectangles, sudoku designs
Volume 7 Issue 5 - 2018

Jambulingam Subramani

Department of Statistics, Pondicherry University, India

Correspondence: Jambulingam Subramani, Department of Statistics, Pondicherry University, RV Nagar, Kalapet, Puducherry-605 014, India, Email drgsubramani@yahoo.co.in, drgsubramani@gmail.com

Received: October 09, 2018 | Published: October 31, 2018

\section{Introduction}

The foremost and an important work to be completed immediately after the bed coffee in the morning by many is to solve a simple puzzle called Sudoku appeared in the newspapers and/or magazines. In fact millions of people from different parts of the world including from Japan, Great Britain, India and elsewhere become addict to tackle the latest edition of the Sudoku Puzzle. The puzzle typically consists of a nine-by-nine grid. Some of the grids contain numbers; most of the grids are blank. The goal is to fill in the blanks with digits from 1 to 9 so that each row, each column, and each of the nine three-bythree squares within the outer squares making up the grid contains just one of each of the nine digits. Here, the rules are very simple but the puzzles can be very challenging and highly addictive. It's basically a logic puzzle; there's no math involved in solving it. The digits could just as easily be nine different letters, shapes, or colors. There is mathematics and computer science, however, in analyzing the puzzles and creating efficient computer programs for generating and solving the Sudoku puzzles. A Sudoku grid is a special case of a mathematical object called a Latin square. A Latin square consists of $n$ sets of numbers from 1 to $n$ arranged in a square pattern so that no row or column contains the same number twice or more. The additional constraint is that a standard nine-by-nine sudoku puzzle has threeby-three squares within the Latin square that also contain each of the nine digits once and only once.

Recently, Subramani \& Ponnuswamy ${ }^{1}$ have introduced Sudoku puzzles as Sudoku square designs, which will extract more sources of variations than the Latin square designs do. Further they have discussed four different variations of the Sudoku square designs together with their construction and analysis. Subramani ${ }^{2}$ has extended the work of Subramani \& Ponnuswamy ${ }^{1}$ and has introduced the construction and analysis of orthogonal Sudoku square designs. For further details on the definition, design, analysis and applications of Sudoku square designs the readers are referred to Bailey et al. ${ }^{3}$ Lorch, ${ }^{4}$ Subramani \& Ponnuswamy ${ }^{1}$ and the references cited therein. For a detailed discussion on the treatment effects, interaction effects, nested factors and their effects, the assumptions, analysis of the new experimental designs to be developed for new situations, one may refer to Cochran and Cox, ${ }^{5}$ Das \& Dey, ${ }^{6}$ Hicks, ${ }^{7}$ and the references cited there in. ${ }^{8-12}$
It is to be noted that the Sudoku square designs are applicable whenever the number of treatments are $\mathrm{m}^{2}$ and the corresponding Sudoku square designs are of orders $\mathrm{m}^{2} \times \mathrm{m}^{2}$. In this paper an attempt is made to include the Sudoku square designs with $\mathrm{mn}$ treatments. However the difference is that the outer Latin square of order $\mathrm{mn} \times \mathrm{mn}$ contains $\mathrm{mn}$ rectangles of order $\mathrm{m} \times \mathrm{n}$. Consequently the resulting Sudoku square designs are called as "Sudoku square designs with rectangles".

The Objective of the present work is

(i) To develop a method to construct Sudoku square designs with rectangles

(ii) To explain various factors involved in the ANOVA techniques

(iii) To develop statistical models and analyze the data from Sudoku square designs with rectangles

(iv) To identify the applications for Sudoku square designs with rectangles together with an numerical example.

\section{Construction of Sudoku square designs with rectangles}

To construct Sudoku square designs with rectangles of orders $\mathrm{mn} \times \mathrm{mn}$ sequentially, follow the steps given below:

Step 1: Write the $m$ numbers from 1 to $m n$ in a matrix of order $\mathrm{m} \times \mathrm{n}$ form sequentially starting from row 1 to row $\mathrm{m}$ as given in Table 1.

Table I Procedure Given in Step I

\begin{tabular}{cccccc}
\hline \multicolumn{6}{c}{ Column } \\
\hline \multirow{4}{*}{ Row } & & I & 2 & 3 & 4 \\
& I & 1 & 2 & 3 & 4 \\
& 2 & 5 & 6 & 7 & 8 \\
& 3 & 9 & 10 & 11 & 12 \\
& 4 & 13 & 14 & 15 & 16 \\
& 5 & 17 & 18 & 19 & 20
\end{tabular}


Step 2: Write the $\mathrm{n}$ columns obtained in step 1, one by one to get a column of order $\mathrm{mn}$ as given in Table 2 .

Step 3: Column 2 can be obtained from column 1 by adding 1 to each of its elements and reduce to mod $\mathrm{mn}$ if it exceeds the value $\mathrm{mn}$. Proceed in the similar way to complete all the columns. The completed Sudoku square design of order 20 is given in Table 3.
The above three steps lead to construct a complete Sudoku square design of any order sequentially. Here, we explain the method of construction for the case of 20 treatments with $m=5$ and $n=4$ in a systematic way.

More examples of Sudoku square designs with rectangles of orders 6,8 and 12 are given below (Figures $1-3$ ):

Table 2 Procedure Given in Step 2

\begin{tabular}{|c|c|c|c|c|c|c|c|c|c|c|c|c|c|c|c|c|c|c|c|c|}
\hline & $I$ & 2 & 3 & 4 & 5 & 6 & 7 & 8 & 9 & 10 & 11 & 12 & 13 & 14 & 15 & 16 & 17 & 18 & 19 & 20 \\
\hline 1 & 1 & & & & & & & & & & & & & & & & & & & \\
\hline 2 & 5 & & & & & & & & & & & & & & & & & & & \\
\hline 3 & 9 & & & & & & & & & & & & & & & & & & & \\
\hline 4 & 13 & & & & & & & & & & & & & & & & & & & \\
\hline 5 & 17 & & & & & & & & & & & & & & & & & & & \\
\hline 6 & 2 & & & & & & & & & & & & & & & & & & & \\
\hline 7 & 6 & & & & & & & & & & & & & & & & & & & \\
\hline 8 & 10 & & & & & & & & & & & & & & & & & & & \\
\hline 9 & 14 & & & & & & & & & & & & & & & & & & & \\
\hline 10 & 18 & & & & & & & & & & & & & & & & & & & \\
\hline Rows & & & & & & & & & & & & & & & & & & & & \\
\hline 11 & 3 & & & & & & & & & & & & & & & & & & & \\
\hline 12 & 7 & & & & & & & & & & & & & & & & & & & \\
\hline 13 & 11 & & & & & & & & & & & & & & & & & & & \\
\hline 14 & 15 & & & & & & & & & & & & & & & & & & & \\
\hline 15 & 19 & & & & & & & & & & & & & & & & & & & \\
\hline 16 & 4 & & & & & & & & & & & & & & & & & & & \\
\hline 17 & 8 & & & & & & & & & & & & & & & & & & & \\
\hline 18 & 12 & & & & & & & & & & & & & & & & & & & \\
\hline 19 & 16 & & & & & & & & & & & & & & & & & & & \\
\hline 20 & 20 & & & & & & & & & & & & & & & & & & & \\
\hline
\end{tabular}


Table 3 Procedure Given in Step 3

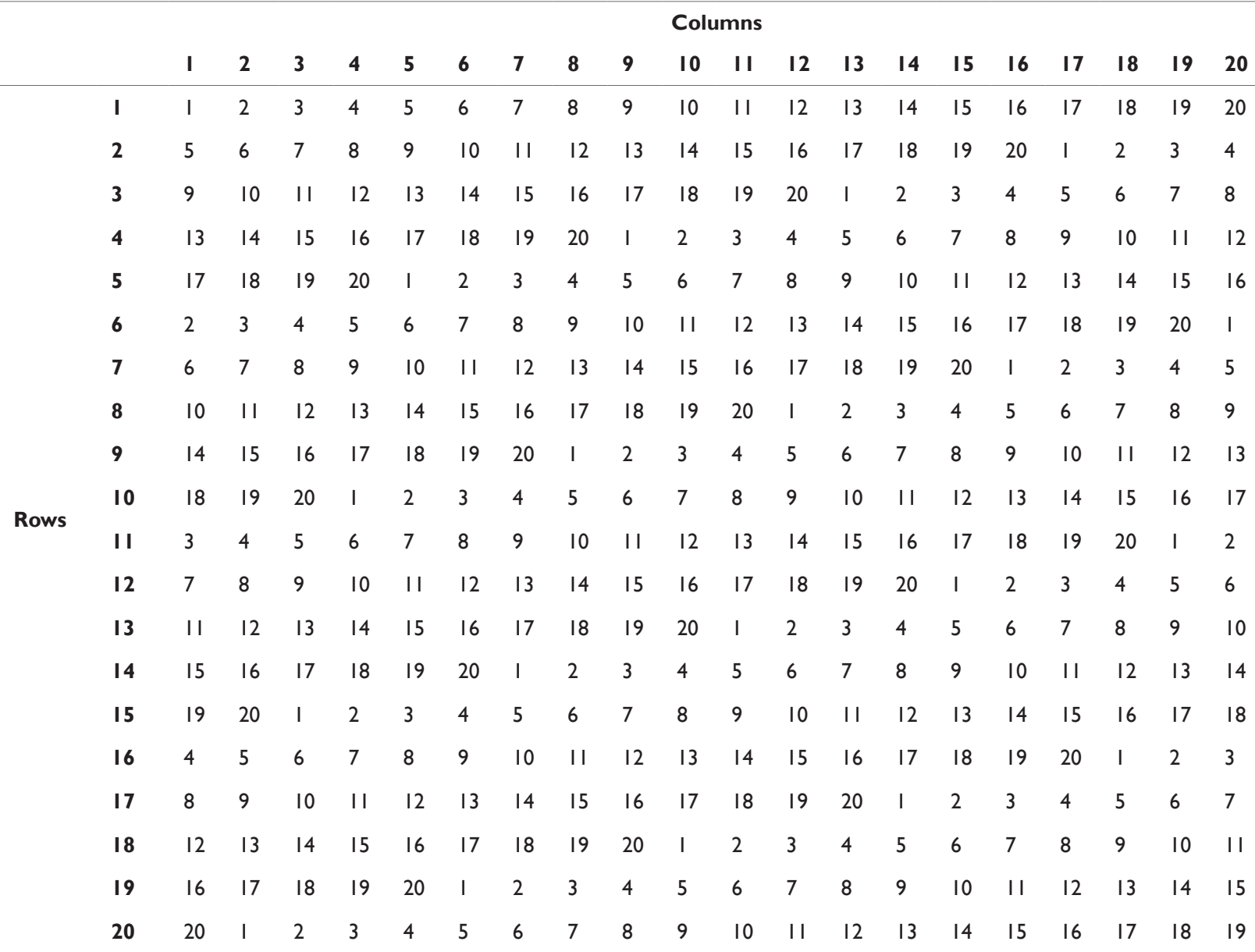

\begin{tabular}{|l|l|l|l|l|l|}
\hline 1 & 2 & 3 & 4 & 5 & 6 \\
\hline 3 & 4 & 5 & 6 & 1 & 2 \\
\hline 5 & 6 & 1 & 2 & 3 & 4 \\
\hline 2 & 3 & 4 & 5 & 6 & 1 \\
\hline 4 & 5 & 6 & 1 & 2 & 3 \\
\hline 6 & 1 & 2 & 3 & 4 & 5 \\
\hline
\end{tabular}

Figure I Sudoku design of order 6.

\begin{tabular}{|l|l|l|l|l|l|l|l|}
\hline 1 & 2 & 3 & 4 & 5 & 6 & 7 & 8 \\
\hline 3 & 4 & 5 & 6 & 7 & 8 & 1 & 2 \\
\hline 5 & 6 & 7 & 8 & 1 & 2 & 3 & 4 \\
\hline 7 & 8 & 1 & 2 & 3 & 4 & 5 & 6 \\
\hline 2 & 3 & 4 & 5 & 6 & 7 & 8 & 1 \\
\hline 4 & 5 & 6 & 7 & 8 & 1 & 2 & 3 \\
\hline 6 & 7 & 8 & 1 & 2 & 3 & 4 & 5 \\
\hline 8 & 1 & 2 & 3 & 4 & 5 & 6 & 7 \\
\hline
\end{tabular}

Figure 2 Sudoku design of order 8. 


\begin{tabular}{|l|l|l|l|l|l|l|l|l|l|l|l|}
\hline 1 & 2 & 3 & 4 & 5 & 6 & 7 & 8 & 9 & 10 & 11 & 12 \\
\hline 4 & 5 & 6 & 7 & 8 & 9 & 10 & 11 & 12 & 1 & 2 & 3 \\
\hline 7 & 8 & 9 & 10 & 11 & 12 & 1 & 2 & 3 & 4 & 5 & 6 \\
\hline 10 & 11 & 12 & 1 & 2 & 3 & 4 & 5 & 6 & 7 & 8 & 9 \\
\hline 2 & 3 & 4 & 5 & 6 & 7 & 8 & 9 & 10 & 11 & 12 & 1 \\
\hline 5 & 6 & 7 & 8 & 9 & 10 & 11 & 12 & 1 & 2 & 3 & 4 \\
\hline 8 & 9 & 10 & 11 & 12 & 1 & 2 & 3 & 4 & 5 & 6 & 7 \\
\hline 11 & 12 & 1 & 2 & 3 & 4 & 5 & 6 & 7 & 8 & 9 & 10 \\
\hline 3 & 4 & 5 & 6 & 7 & 8 & 9 & 10 & 11 & 12 & 1 & 2 \\
\hline 6 & 7 & 8 & 9 & 10 & 11 & 12 & 1 & 2 & 3 & 4 & 5 \\
\hline 9 & 10 & 11 & 12 & 1 & 2 & 3 & 4 & 5 & 6 & 7 & 8 \\
\hline 12 & 1 & 2 & 3 & 4 & 5 & 6 & 7 & 8 & 9 & 10 & 11 \\
\hline
\end{tabular}

Figure 3 Sudoku design of order 12.

\section{Sudoku designs modeling and analysis}

In section 2, we have presented a sequential method of constructing Sudoku square designs with rectangles of any order. Now by keeping the successive $\mathrm{m}$ rows ( $\mathrm{n}$ columns) as blocks (treatments) of a randomized block design with $\mathrm{mn}$ observations in each cell and the outer Sudoku square as a Latin square design of order mn, one can construct a Sudoku square experimental design with rectangles. These Sudoku designs are viewed like Latin square designs, F-square designs and replicated Latin square designs. One of the important properties of any experimental design is the "Randomization Principle". One has to maintain the randomization to achieve unbiased results. The randomness can be achieved by selecting any one of the available completed Sudoku grids or constructing the designs by using the sequential method discussed in Section 2 as done normally in the case of Latin square designs. Randomly arranging the $\mathrm{mn}$ numbers, one may get $(\mathrm{mn})$ ! distinct arrangements of the numbers, which will lead to $(\mathrm{mn})$ ! distinct Sudoku square designs, which will also ensure the randomization of the treatments. Further by interchanging the rows or columns within the row blocks or column blocks one may get additional Sudoku square designs with rectangles.

The Sudoku square design with rectangles of order $\mathrm{mn}$ and its Analysis of Variance (ANOVA) model together with the various assumptions and the ANOVA table are given below:

$$
\mathrm{Y}_{\mathrm{lp}(\mathrm{ijk})}=\mu+\alpha_{\mathrm{i}}+\beta_{\mathrm{j}}+\alpha \beta_{\mathrm{ij}}+\mathrm{r}_{1}+\mathrm{c}_{\mathrm{p}}+\tau_{\mathrm{k}}+\mathrm{e}_{\mathrm{ij}(\mathrm{klpq})} \begin{aligned}
& \mathrm{i}=1,2, \ldots, \mathrm{n}, \mathrm{j}=1,2, \ldots, \mathrm{m} \\
& \text { and } \mathrm{k}, 1, \mathrm{p}=1,2, \ldots, \mathrm{mn}
\end{aligned}
$$

Where $\mu=$ General mean effect

$\alpha_{\mathrm{i}}=\mathrm{i}^{\text {th }}$ Row (RBD) effect

$\beta_{\mathrm{j}}=\mathrm{j}^{\text {th }}$ Column (RBD) effect

$\alpha \beta_{\mathrm{ij}}=\mathrm{ij}^{\text {th }}$ Interaction (RBD) effect

$r_{1}=1^{\text {th }}$ Row (LSD) effect

$c_{p}=p^{\text {th }}$ Column (LSD) effect

$\tau_{\mathrm{k}}=\mathrm{k}^{\text {th }}$ Treatment (LSD) effect

$\mathrm{e}_{\mathrm{lp}}=$ Error component with mean zero and variance $\sigma^{2}$

The Sudoku square design with rectangles consists of randomized block design with $\mathrm{m}$ blocks (columns) and $\mathrm{n}$ treatments (rows) with $\mathrm{mn}$ observations in each cell and a Latin square design with $\mathrm{mn}$ rows, columns and treatments as given below Table 4 \& Table 5 .

After a little algebra we have obtained the formulae for computing various sum of squares and degrees of freedom and formed the Analysis of Variance Table given in Table 6.

Where

$$
\begin{aligned}
& \text { TSS }=\sum_{\mathrm{l}=1}^{\mathrm{N}} \sum_{\mathrm{p}=1}^{\mathrm{N}} \mathrm{Y}_{\mathrm{lp}}^{2}-\frac{\mathrm{G}^{2}}{\mathrm{~N}^{2}} \\
& \mathrm{G}=\sum_{\mathrm{l}=1}^{\mathrm{N}} \sum_{\mathrm{p}=1}^{\mathrm{N}} \mathrm{Y}_{\mathrm{ij}} \text { and } \mathrm{N}=\mathrm{mn} \\
& \mathrm{SSRR}=\sum_{\mathrm{i}=1}^{\mathrm{n}} \frac{\mathrm{RB}_{\mathrm{i..}}^{2}}{\mathrm{mN}}-\frac{\mathrm{G}^{2}}{\mathrm{~N}^{2}}
\end{aligned}
$$




$$
\begin{aligned}
& \operatorname{SSCR}=\sum_{j=1}^{m} \frac{C B_{j .}^{2}}{n N}-\frac{G^{2}}{N^{2}} \\
& \text { SSRCR }=\sum_{i=1}^{n} \sum_{j=1}^{m} \frac{R C_{i j}^{2}}{N}-\sum_{i=1}^{n} \frac{R B_{i . .}^{2}}{m N}-\sum_{j=1}^{m} \frac{C B_{j . .}^{2}}{n N}+\frac{G^{2}}{N^{2}} \\
& S S R L=\sum_{i=1}^{N} \frac{R L_{1}^{2}}{N}-\frac{G^{2}}{N^{2}}
\end{aligned}
$$$$
\mathrm{SSCL}=\sum_{\mathrm{p}=1}^{\mathrm{N}} \frac{\mathrm{CL}_{\mathrm{p}}^{2}}{\mathrm{~N}}-\frac{\mathrm{G}^{2}}{\mathrm{~N}^{2}}
$$$$
\operatorname{SSTL}=\sum_{\mathrm{k}=1}^{\mathrm{N}} \frac{\mathrm{TL}_{\mathrm{k}}^{2}}{\mathrm{~N}}-\frac{\mathrm{G}^{2}}{\mathrm{~N}^{2}}
$$$$
\mathrm{ESS}=\sum_{\mathrm{l}=1}^{\mathrm{N}} \sum_{\mathrm{p}=1}^{\mathrm{N}} \mathrm{Y}_{\mathrm{lp}}^{2}-\sum_{\mathrm{i}=1}^{\mathrm{n}} \sum_{\mathrm{j}=1}^{\mathrm{m}} \frac{\mathrm{RC}_{\mathrm{ij}}^{2}}{\mathrm{~m}^{3}}-\sum_{\mathrm{l}=1}^{\mathrm{N}} \frac{\mathrm{RL}_{1}^{2}}{\mathrm{~N}}-\sum_{\mathrm{p}=1}^{\mathrm{N}} \frac{\mathrm{CL}_{\mathrm{p}}^{2}}{\mathrm{~N}}-\sum_{\mathrm{k}=1}^{\mathrm{N}} \frac{\mathrm{TL}_{\mathrm{k}}^{2}}{\mathrm{~N}}+\frac{3 \mathrm{G}^{2}}{\mathrm{~N}^{2}}
$$

Table 4 Sudoku square design of order $\mathrm{n} \times \mathrm{m}-\mathrm{RBD}$ part

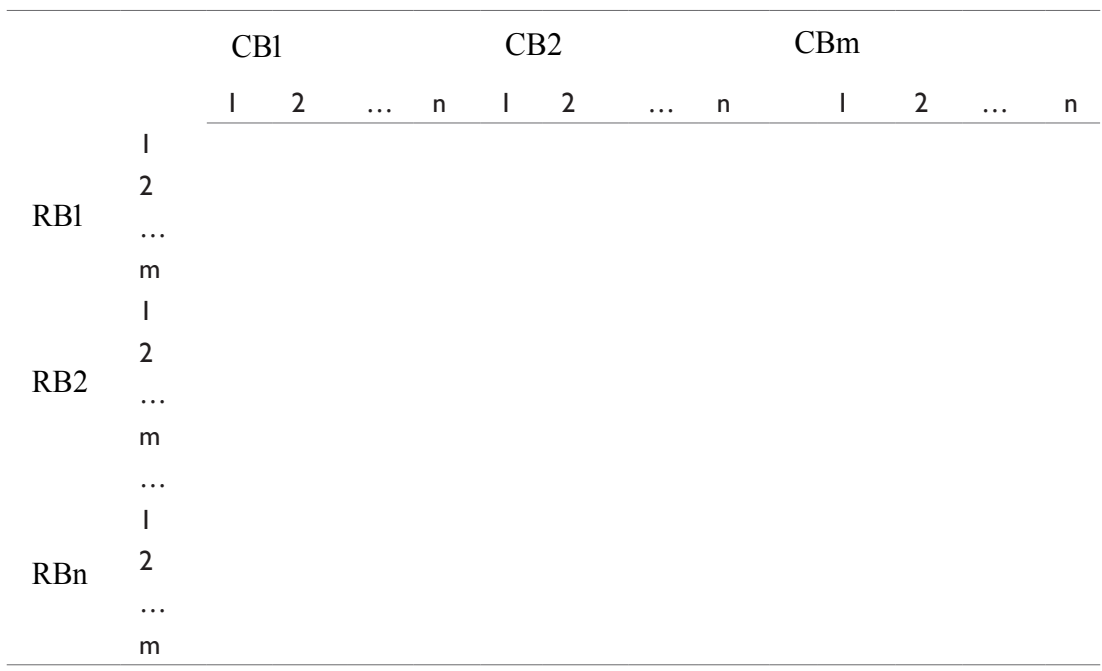

Table 5 Sudoku square design of order mn - LSD part

\begin{tabular}{|c|c|c|c|c|}
\hline Source & $\begin{array}{l}\text { Sum of } \\
\text { squares }\end{array}$ & $\begin{array}{l}\text { Degrees of } \\
\text { freedom }\end{array}$ & $\begin{array}{l}\text { Mean } \\
\text { squares }\end{array}$ & $\begin{array}{l}\text { F-Ratio } \\
\text { (Observed) }\end{array}$ \\
\hline Row (RBD) & SSRR & $(n-I)$ & $M S R R=S S R R / d f$ & MSRR/MSE \\
\hline Column (RBD) & SSCR & $(\mathrm{m}-\mathrm{I})$ & $M S C R=S S C R / d f$ & MSCR/MSE \\
\hline RC-interaction (RBD) & SSRCR & $(m-I)(n-I)$ & $\mathrm{MSRCR}=\mathrm{SSRCR} / \mathrm{df}$ & MSRCR/MSE \\
\hline ROWS (LSD) & SSRL & $(m-I)(n-I)$ & $M S R L=S S R L / d f$ & MSRL/MSE \\
\hline COLUMNS (LSD) & SSCL & $(\mathrm{mn}-\mathrm{I})$ & $M S C L=S S C L / d f$ & MSCL/MSE \\
\hline Treatments (LSD) & SSTL & $(m n-I)$ & $M S T L=S S T L / d f$ & MSTL/MSE \\
\hline ERROR & SSE & $(m n-I)(m n-3)$ & $\mathrm{MSE}=\mathrm{SSE} / \mathrm{df}$ & --- \\
\hline TOTAL & TSS & $m^{2} n^{2}-1$ & --- & -.- \\
\hline
\end{tabular}

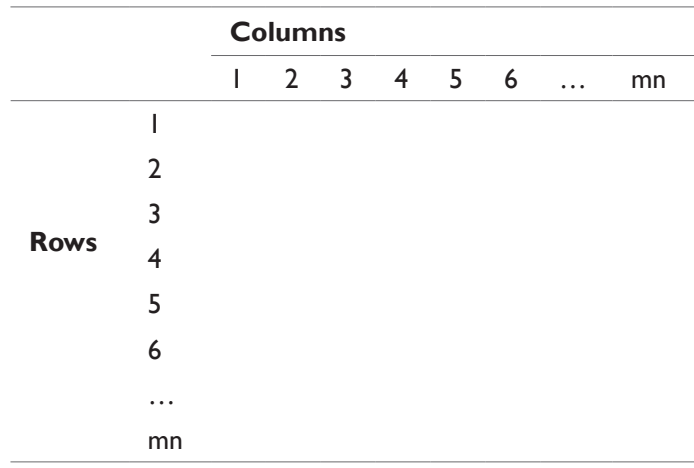

Table 6 ANOVA Table of Sudoku Design of order mn 


\section{Numerical example}

In this section the analysis of Sudoku square design with rectangles is explained with the help of a numerical example. For this purpose we have generated hypothetical data and are given below: (Table 7).

The various sums of squares are obtained from the data given above for the Sudoku design and are presented in the following ANOVA table. The statistical inferences drawn from the ANOVA table are also given immediately after the ANOVA table (Table 8).

From the above ANOVA Table, it can be observed that the F-Ratio of observed values is lesser than the respective expected value obtained from the F-table at 5\% level of significance. Hence it is concluded that none of the effect is significant at $5 \%$ level of significance.

Table 7 Hypothetical data of a Sudoku square design of order 12

\begin{tabular}{|c|c|c|c|c|c|c|c|c|c|c|c|c|c|}
\hline & & \multicolumn{3}{|c|}{ Column RBD-I } & \multicolumn{3}{|c|}{ Column RBD-2 } & \multicolumn{3}{|c|}{ Column RBD-3 } & \multicolumn{3}{|c|}{ Column RBD-4 } \\
\hline & & $\mathrm{CI}$ & C2 & C3 & $\mathrm{C4}$ & C5 & C6 & C7 & C8 & C9 & CIO & CII & $\mathrm{C} 12$ \\
\hline \multirow{8}{*}{ Row RBD-I } & RI & I & 2 & 3 & 4 & 5 & 6 & 7 & 8 & 9 & 10 & 11 & 12 \\
\hline & & (I5) & (II) & $(16)$ & (2I) & (22) & $(21)$ & $(14)$ & (19) & (I5) & $(16)$ & (23) & (22) \\
\hline & $\mathrm{R} 2$ & 4 & 5 & 6 & 7 & 8 & 9 & 10 & 11 & 12 & I & 2 & 3 \\
\hline & & $(18)$ & (23) & (20) & (18) & (20) & (23) & $(17)$ & $(22)$ & (I5) & (16) & (23) & (22) \\
\hline & R3 & 7 & 8 & 9 & 10 & 11 & 12 & I & 2 & 3 & 4 & 5 & 6 \\
\hline & & (I5) & $(10)$ & $(22)$ & (15) & (18) & (19) & $(24$ & $(15$ & $(16$ & $(18$ & (15 & $(16$ \\
\hline & R4 & 10 & 11 & 12 & I & 2 & 3 & 4 & 5 & 6 & 7 & 8 & 9 \\
\hline & & $(22)$ & $(16)$ & $(23)$ & (22) & (19) & (25) & $(20)$ & $(25)$ & $(12)$ & $(22)$ & $(21)$ & (14) \\
\hline \multirow{8}{*}{$\begin{array}{l}\text { Row } \\
\text { RBD-2 }\end{array}$} & R5 & 2 & 3 & 4 & 5 & 6 & 7 & 8 & 9 & 10 & 11 & 12 & I \\
\hline & & $(17)$ & $(23)$ & (I8) & (15) & (16) & $(21)$ & (I5) & $(I 2)$ & $(16)$ & (16) & $(23)$ & (22) \\
\hline & R6 & 5 & 6 & 7 & 8 & 9 & 10 & 11 & 12 & I & 2 & 3 & 4 \\
\hline & & (20) & $(25)$ & $(12)$ & (26) & (2I) & (17) & $(18)$ & $(25)$ & (19) & (22) & $(2 I)$ & (14) \\
\hline & $\mathrm{R} 7$ & 8 & 9 & 10 & 11 & 12 & I & 2 & 3 & 4 & 5 & 6 & 7 \\
\hline & & $(2 I)$ & (I7) & (I8) & (22) & (16) & (23) & (22) & (19) & $(25)$ & (15) & $(12)$ & (16) \\
\hline & $\mathrm{R} 8$ & 11 & 12 & I & 2 & 3 & 4 & 5 & 6 & 7 & 8 & 9 & 10 \\
\hline & & $(13)$ & $(22)$ & $(2 \mathrm{I})$ & (22) & (20) & (19) & $(14)$ & $(14)$ & $(17)$ & (II) & $(16)$ & (18) \\
\hline \multirow{8}{*}{ Row RBD-3 } & R9 & 3 & 4 & 5 & 6 & 7 & 8 & 9 & 10 & 11 & 12 & I & 2 \\
\hline & & $(12)$ & $(25)$ & $(20)$ & (19) & (22) & (16) & (19) & (I3) & (I8) & (15) & $(12$ & (16) \\
\hline & $\mathrm{R} 10$ & 6 & 7 & 8 & 9 & 10 & 11 & 12 & I & 2 & 3 & 4 & 5 \\
\hline & & $(21)$ & $(22)$ & $(13)$ & (22) & (2I) & (22) & $(20)$ & (19) & $(14)$ & (23) & $(22$ & (19) \\
\hline & RII & 9 & 10 & 11 & 12 & I & 2 & 3 & 4 & 5 & 6 & 7 & 8 \\
\hline & & $(14)$ & $(17)$ & (II) & (16) & (18) & (23) & (I5) & (23) & (16) & (17) & (18) & (22) \\
\hline & $\mathrm{R} / 2$ & 12 & I & 2 & 3 & 4 & 5 & 6 & 7 & 8 & 9 & 10 & 11 \\
\hline & & $(2 \mathrm{I})$ & (22) & (20) & (19) & (I5) & (I2) & (16) & $(2 \mathrm{I})$ & (22) & (20) & (19) & (15) \\
\hline
\end{tabular}

Table 8 ANOVA Table of Sudoku square design with rectangles of order 12

\begin{tabular}{llllll}
\hline Source & $\begin{array}{l}\text { Sum of } \\
\text { squares }\end{array}$ & $\begin{array}{l}\text { Degrees of } \\
\text { freedom }\end{array}$ & $\begin{array}{l}\text { Mean } \\
\text { squares }\end{array}$ & $\begin{array}{l}\text { F-Ratio } \\
\text { (Observed) }\end{array}$ & $\begin{array}{l}\text { Table value* } \\
\text { (Expected) 5\% level }\end{array}$ \\
\hline Rows (RBD) & 3.7639 & 2 & 1.8819 & 0.1267 & 3 \\
Columns (RBD) & 63.6667 & 3 & 21.2222 & 1.4286 & 2.6 \\
Row-Column Interaction (RBD) & 41.7917 & 6 & 6.96528 & 0.4689 & 2.1 \\
Rows (LSD) & 187.3889 & 11 & 17.0354 & 1.1468 & 1.75 \\
Columns (LSD) & 126.5556 & 11 & 11.5051 & 0.7745 & 1.75 \\
Treatments (LSD) & 102.0556 & 11 & 9.2778 & 0.6246 & 1.75 \\
ERROR & 1470.667 & 99 & 14.8552 & & \\
& & & & &
\end{tabular}

*The values are taken with degrees of freedom $(*, \infty)$ instead of $(*, 99)$ from the F-Table 


\section{Application of Sudoku square designs with rectangles}

One can easily find applications of Sudoku designs with rectangles in real life situations. For example, in the agricultural experiments, suppose one may be interested in developing hybrid seeds or identifying the variety of seeds among several seeds available, which will give better yields. To conduct an experimental study for comparing $k$ variety of seeds (factors), one must have $\mathrm{kr}$ homogeneous plots (experimental units), where $r$ is the number of replications of each variety of seeds. Assume that if $\mathrm{kr}$ homogeneous plots are available then one must use the completely randomized design for collecting the statistical data and use the one-way ANOVA for analyzing the data. The total variation is divided into two factors pertaining to the error component and to the seeds (factor A).

Now let us assume that the kr plots are not homogeneous. Further assume that the $\mathrm{kr}$ plots are grouped into $\mathrm{k}$ sub groups of size $r$ such that the plots within each group are homogeneous and between the groups they are heterogeneous. For example, the plots within the group are having the same soil fertility and between the groups are having different soil fertility. The variety of seeds form one factor and the different soil fertilities form another factor, which need the randomized block designs to collect the statistical data and the twoway ANOVA for analyzing the data. The total variation is divided into three pertaining to the error component; due to the seeds (factor A) and due to the soil fertilities (factor B).

Similarly, if one has a set of different fertilizers, in addition to the different variety of seeds and soil fertilities then Latin square designs have to be used provided the number of seeds, soil fertilities and the fertilizers are the same. The total variation is divided into four pertaining to the error component; due to the seeds (factor A); due to the soil fertilities (factor B) and due to the fertilizers (factor C).

Suppose that one wants to use more number of different factors, which are influencing the effects of the main factor (in this case the variety of seeds) one has to use a more advanced statistical designs like Graeco Latin squares designs, replicated Latin squares designs etc. For example, one wants to compare the performance or effects of different variety of seeds by incorporating different levels of water quality; different methods of cultivation; different types of natural fertilizers; different types of artificial fertilizers; and different types of soil fertilities; the existing statistical designs mentioned above are not useful. However one can use the proposed Sudoku designs with rectangles by studying the effects of different variety of seeds by incorporating all the factors given above as explained below:

Consider the variety of Seeds as different treatments; different levels of Water Quality as Rows of RBD; different methods of cultivation as Columns of RBD; different type of fertilizers (Artificial) as Rows of LSD; different type of fertilizers (Natural) as different
Columns of LSD; different levels of Soil Fertility as treatments of LSD. Sudoku designs with rectangles and their statistical models together with the analysis are given earlier. Similarly one can look for applications of Sudoku square designs with rectangles in the fields like genetical statistics, biostatistics, medical statistics and animal breeding experiments etc. The authors are planned to study in detail about other variants of Sudoku square designs with rectangles and various optimality criteria in their future projects.

\section{Acknowledgements}

The author has expressed his gratitude's and thanks to the editor and the reviewer for their constructive comments which has enhanced the presentation of the paper in a more meaningful way.

\section{Conflict of interest}

Author declares that there is no conflict of interest.

\section{References}

1. MS Virk, AK Mahal. On balancing in repeated measurements designs Model Assisted Statistics and Applications. 2006;1:77-81.

2. J Subramani, KN Ponnuswamy. Construction and analysis of Sudoku designs. Model Assisted Statistics and Applications. 2009;4:287-301.

3. R Bailey P, Cameron, R Connelly. Sudoku, gerechte designs, resolutions, affine space, spreads, reguli, and Hamming codes. Amer Math Monthly. 2008; $115: 383-403$

4. J Lorch. Mutually orthogonal families of linear Sudoku solutions. $J$ Austral Math Soc. 2009;87:409-420.

5. WG Cochran, GM Cox. Experimental Designs. 2nd edn. New York: John Wiley and Sons; 1957.

6. Das, A Dey. A Note on Construction of Graeco Latin Square of order $2 n+1$. J Indian Soc Agric Statist. 1890;42:247-249.

7. CR Hicks. Fundamental Concepts in the Design of Experiments. New York: Holt, Rinehart and Winston; 1964

8. Design and Analysis of Experiments. 2nd edn. New York: John Wiley and Sons; 1984

9. VK Sharma C, Varghese, S Jaggi. Tetrahedral and cubical association schemes with related PBIB(3) designs. Model Assisted Statistics and Applications. 2010;5:93-99.

10. J Subramani. A Further Note on Construction of Graeco Latin Square of order 2n+1. J Indian Soc Agric Statist. 1996;48:356-359.

11. J Subramani. Construction and analysis of orthogonal (Graeco) Sudoku square esigns. Model Assisted Statistics and Applications. 2013;8(3):239 246.

12. MS Virk, AK Mahal. Some balanced repeated measurements designs. Model Assisted Statistics and Applications. 2007;2:37-39. 